\title{
Cochlear Implantation Surgery in Jervell and Lange-Nielsen Syndrome Patients: Our Experience
}

\author{
Rajashree D Godbole ${ }^{1}$, Joyshankar Jana ${ }^{2}$, Prithviraj Gauri ${ }^{3}$, Amol B Mhetre $^{4}$
}

\begin{abstract}
Jervell and Lange-Nielsen syndrome represents a rare autosomal recessive cause of congenital deafness. The affected patients have prolongation of QT interval on electrocardiogram. These patients may present with cardiac arrhythmias, syncopal episodes, and predisposition to sudden death. These patients are predisposed to polymorphic ventricular tachycardia-torsades de pointes leading to syncope and sudden death. Surgical stress in the perioperative period, anxiety, loud sounds, and fear may trigger fatal arrhythmias. We are reporting anesthetic management of two pediatric patients posted for cochlear implantation surgery.

Keywords: Cardiac arrhythmias, Jervell and Lange-Nielsen syndrome, Prolonged QT interval, Torsades de pointes.

The Journal of Medical Sciences (2019): 10.5005/jp-journals-10045-00133
\end{abstract}

\section{INTRODUCTION}

Long QT syndrome (LQTS) is a genetic abnormality of ventricular repolarization. Congenital LQTS results from mutation in the genes encoding cardiac ion channels. Acquired LQTS is caused by drugs or metabolic abnormalities. The age of presentation in congenital LQTS may vary from in utero to adulthood. ${ }^{1}$ Majority of the events occurring in patients of LQTS are related to physical activity, excitement, and emotional stress.' All patients of LQTS are not symptomatic. Many times LQTS patients are misdiagnosed and treated for epilepsy. Family history of sudden death may be present, but family history of sensorineural deafness is extremely uncommon. The morbidity and mortality are very high in patients with LQTS because of abnormal cardiac repolarization and prolonged QT interval. The anesthesiologists should be aware of necessary interventions and treatment modalities for LQTS patients.

\section{Case Description}

A 2-year-old male baby weighing $9.5 \mathrm{~kg}$ posted for cochlear implant surgery was investigated preoperatively. The routine preoperative investigations done in patients of cochlear implantation surgery in our institute are hemogram, urine routine examination, and electrocardiogram (ECG). His ECG-corrected QT interval (QTc) was $480 \mathrm{~ms}$. The child was asymptomatic till date. This was an accidental finding. This child was not born from consanguineous marriage.

The second baby was 6-year-old female weighing $16 \mathrm{~kg}$. She was posted for cochlear implantation surgery. She had history of convulsions since 2 years and was on antiepileptic treatment. On treatment, she was having convulsions in-between. In her preoperative ECG, QTc was 580 ms. This child was born from consanguineous marriage. Both the children were diagnosed LQTS. Both of them were referred to pediatric cardiologist to rule out other causes of LQTS. Serum electrolytes, serum calcium, and serum magnesium levels were measured which were normal in both the children. In both the families, there was no family history of sudden death or congenital deafness. Congenital deafness with LQTS confirmed the diagnosis of JLNS in both the children. They were started on tab propranolol $1 \mathrm{mg} / \mathrm{kg}$ preoperatively. The second child was taking antiepileptic treatment which was
${ }^{1-4}$ Department of Anaesthesiology, King Edward Memorial Hospital, Pune, Maharashtra, India

Corresponding Author: Rajashree D Godbole, Department of Anaesthesiology, King Edward Memorial Hospital, Pune, Maharashtra, India, Phone: +91 9822049748, e-mail: rajashree.godbole@gmail.com How to cite this article: Godbole RD, Jana J, Gauri P, et al. Cochlear Implantation Surgery in Jervell and Lange-Nielsen Syndrome Patients: Our Experience. J Med Sci 2019;5(4):96-98.

Source of support: Nil

Conflict of interest: None

continued. After optimization by the cardiologist, the babies were posted for surgery.

The babies were kept nil by mouth for 2 hours for clear fluids and 6 hours for solids.

The $\beta$-blocker was continued on the day of surgery. The second baby was administered her morning dose of sodium valproate. Midazolam $0.5 \mathrm{mg} / \mathrm{kg}$ was administered orally to avoid sympathetic over activity due to crying, preoperative stress. Defibrillator pads and pacing equipment were kept ready.

Eutectic mixture of local anesthetics (EMLA) cream was applied to the hand on the opposite side of the ear to be operated 45 minutes before induction of anesthesia. Intravenous line was secured in the mother's lap and then the baby was taken inside the pediatric operation room.

Electrocardiogram leads, noninvasive blood pressure monitor, and pulse oximeter probe were attached for intraoperative monitoring. The defibrillator pads were attached on the chest of the patient anteroposteriorly, and defibrillator was kept on pacing mode.

Anesthesia was induced by administering injection fentanyl $2 \mu \mathrm{g} / \mathrm{kg}$, injection propofol $2 / \mathrm{kg}$, and paralyzed with injection Vecuronium bromide $0.1 \mathrm{mg} / \mathrm{kg}$. Intubation was performed with uncuffed endotracheal tube. End tidal $\mathrm{CO}_{2}$ and temperature probes were connected. We ventilated both the patients with Datex Fabius Plus machine using intermittent positive-pressure ventilation mode with a tidal volume of $10 \mathrm{~mL} / \mathrm{kg}$. Pediatric circuits were used for 
both the patients. Inj cefurixime $50 \mathrm{mg} / \mathrm{kg}$ was administered on the table after induction as antibiotic to prevent infection, acute otitis media, meningitis, and loss of implant.

Anesthesia was maintained with $\mathrm{O}_{2}+\mathrm{N}_{2} \mathrm{O}$ along with intravenous fentanyl $0.5 \mu \mathrm{g} / \mathrm{kg}$ per hour and propofol $0.5 \mathrm{mg} /$ $\mathrm{kg} / \mathrm{hour}$ by infusion. Magnesium sulfate infusion $30 \mathrm{mg} / \mathrm{kg}$ was administered over 30 minutes after induction of anesthesia to avoid intraoperative cardiac arrhythmias. Injection Dexamethasone 0.1 $\mathrm{mg} / \mathrm{kg}$ IV was administered as an antiemetic. The neuromuscular blockade was not reversed at the end of the surgery. Spontaneous recovery was allowed, and the patients were extubated. Cefuroxime $250 \mathrm{mg} 8$ hourly was continued orally for 5 days.

Postoperatively, the children were monitored in a quiet environment with defibrillator pads attached. Injection paracetamol $15 \mathrm{mg} / \mathrm{kg}$ was administered for postoperative pain relief and continued 8 hourly postoperatively. In both the patients, surgery and the postoperative period were uneventful. Both the children were discharged on day 2 of surgery with $\beta$-blocker treatment.

Following were avoided: pre-, intra-, and postoperatively sympathetic over activity, hypokalemia, hypomagnesaemia, hypocalcemia, hypothermia, and hypoxia. Drugs like inhalational anesthetic agents can prolong QT interval and were avoided during anesthesia. Ondansetron more than $4 \mathrm{mg}$, anticholinergic drugs, and anticholinesterases can prolong QT interval and hence were avoided.

\section{Discussion}

Anton Jervell and Fred Lange-Nielsen first described the electrical conduction defect in the heart called LQTS in 1957. ${ }^{2}$ Jervell LangeNielsen Syndrome (JLNS) is an inherited autosomal recessive disease characterized by a congenital bilateral deafness with QT prolongation on ECG. A potassium channel gene is affected causing recessive JLNS and dominant Romano Ward syndrome with no deafness and only prolongation of QT. ${ }^{3,4}$ Romano Ward syndrome depends on mutations affecting at least five genes encoding sodium and potassium channels. JLNS is autosomal recessive which depends on homozygous or compound heterozygous mutations on either one of the two genes-KCNQ1 or KCNE1. ${ }^{5}$ KCNQ1 geneaffected patients are at higher risk for arrhythmogenic events compared to KCNE1 mutation.

Consanguineous marriage increases the risk to have children with a recessive genetic disorder. The risk for two carrier parents to both pass the defective gene and have an affected child is $25 \%$ with each pregnancy. ${ }^{6}$ Our second patient was a child born from consanguineous marriage.

Triggers for cardiac events are exercise, emotions in 95\% times, and rest and sleep in $7 \%$ times. Swimming induces cardiac events $16 \%$ times. ${ }^{4,5,7}$

Ten percent patients become symptomatic in first year of life, $50 \%$ patients in the third year, and $90 \%$ around 18 years of age. ${ }^{5}$

Females are at lower risk of life-threatening events excluding syncope. ${ }^{4,6}$ QTc interval is a major risk factor for patients to become symptomatic or remain asymptomatic. Two percent patients who remain truly asymptomatic after 15 years of age have QTc interval around $488 \mathrm{~ms}$ which is significantly shorter than symptomatic patients having QTc around 560 ms. ${ }^{4,5}$ Our first patient was asymptomatic having QTc interval $480 \mathrm{~ms}$, and the second was symptomatic having QTc interval $560 \mathrm{~ms}$.

Bazett formula is used for calculation of heart rate corrected QT interval (QTC). ${ }^{1}$ QTC $=$ QT measured $/ \sqrt{R R}$ (in ECG lead II and V5)
Before making a diagnosis of LQTS, factors prolonging QT interval like hypothermia, hypoxia, hypomagnesemia, hypocalcemia, hypokalemia, and medications should be ruled out. ${ }^{2,3}$

Beta-blockers are the therapy of choice. Patients on betablockers are associated with significant decrease in cardiac events in JLNS. Syncope, aborted cardiac arrest, and LQTS-related deaths continue to occur while patients are on beta-blockers, particularly those who were symptomatic before starting beta blocker therapy. ${ }^{8}$

Nadolol, metoprolol, and propranolol are effective in the treatment of JLNS. Propranolol shows greater shortening of QTC compared to others. ${ }^{4,8}$

Implantable cardioverter defibrillator can be implanted in patients who continue to have syncopal attacks on treatment with beta-blocker, symptomatic children with cardiac events, and patients with torsades de pointes. ${ }^{4,5}$

Left cervical sympathectomy may help some patients but may not be useful in others. ${ }^{9}$ Cervical sympathectomy may not be able to correct the cardiac condition in all the patients with JLNS. ${ }^{9}$ It has been observed that there is reduction in QT dispersion and incidence of torsades de pointes (TdP)., ${ }^{7} 10$

Intraoperative TdP can be treated with injection magnesium sulphate $30 \mathrm{mg} / \mathrm{kg}$ bolus followed by $2-4 \mathrm{mg} / \mathrm{kg}$ infusion. This can effectively terminate and prevent TdP even in the setting of normal Mg levels. ${ }^{7}$ Magnesium sulfate acts as calcium channel blocker, and it activates sodium potassium adenosine triphosphatase in the cell membrane promoting the resting repolarization leading to reduction in rate of arrhythmias. ${ }^{7}$ If magnesium sulfate is ineffective, defibrillation with $2 \mathrm{~J} / \mathrm{kg}$ will be used to terminate TdP. ${ }^{4}$

Postoperatively, the patients were monitored in a quiet environment with defibrillator pads attached to the patient's chest. Adequate postoperative analgesia with Injection paracetamol 15 $\mathrm{mg} / \mathrm{kg}$ was continued eight hourly. Antiemetics like ondansetron and domperidone were avoided.

\section{Conclusion}

In congenitally deaf patients posted for cochlear implantation surgery, preoperative ECG is mandatory to rule out JLNS. Betablocker is the choice of treatment and should be continued on the day of surgery. Defibrillator pads, pacing equipment, and inj. magnesium sulfate must be ready in the operation rooms. We should provide adequate premedication, perioperative analgesia, and careful use of anesthetic and other drugs to avoid QT prolongation. Total intravenous anesthesia with propofol and fentanyl infusion is recommended for maintenance of anesthesia. It is preferable to allow spontaneous recovery from neuromuscular blockade. Postoperatively monitor the patient in quiet environment with good postoperative analgesia.

We feel cochlear implantation can be performed relatively safely in these patients if necessary precautions have been taken appropriately and their auditory outcome is good. Cardiac events that can trigger a life-threatening situation should be avoided throughout their life time. Further research is required to know other variants of such a disease and the implications of the drugs used in the perioperative period.

\section{References}

1. Booker PD, Whyte SD, Laudsans EJ. Long QT syndrome and anaesthesia. Br J Anaesth 2003;90(3):349-366. DOI: 10.1093/bja/ aeg061. 
2. Jervell A. Fred Lange-Nielsen. congenital deaf mutism, functional heart disease with prolongation of QT interval and sudden death. Am Heart J 1957;54(1):59-68. DOI: 10.1016/0002-8703(57) 90079-0.

3. Neyroud N, Tesson F, Denjoy l, et al. A novel mutation in the potassium channel gene KVLQ1 causes the JLN cardioauditory syndrome. Nat Genet 1997;15(2):186-189. DOI: 10.1038/ng0297-186.

4. O'Hare M, Maldonado Y, Munro J. Perioperative management of patients with congenital or acquired disorder of the QT interval. $\mathrm{Br} J$ Anaesth 2018;120(4):629-644. DOI: 10.1016/j.bja.2017.12.040.

5. Schwartz PJ, Spazzolinii C, Crotti L, et al. The JLN syndrome: natural history, molecular basis and clinical outcome. Circulation 2006;113:783-790. DOI: 10.1161/CIRCULATIONAHA.105.592899.

6. National Organization for Rare Disorders. Jervell and Lange-Nielsen Syndrome. rarediseases.org.
7. Kies SJ, Pabelick CM, Hurley HA, et al. Anaesthesia for children with long QT syndrome. Anaesthesiology 2005;102(1):204-210. DOI: 10.1097/00000542-200501000-00029.

8. Moss AJ, Zereba W, Hall WJ, et al. Effectiveness and limitations of beta blocker therapy in congenital long QT syndrome. Circulation 2000;101(6):616-623. DOI: 10.1161/01.CIR.101.6.616.

9. Roy PM, Khanna S, Mehta Y, et al. Anaesthesia management of a case of Jervell Lange-Nielsen syndrome for minimally invasive bilateral thoracoscopic cervicothoracic sympathectomy. Indian J Anaesth 2016;60(6):424-426. DOI: 10.4103/0019-5049.183390.

10. Nair AS, Nirale AM, Gopal TV, et al. A child with Jervell LangeNielsen syndrome for permanent pacemaker implantation and sympathectomy: anaesthesia management and considerations. J Anaesthesiol Clin Pharmacol 2015;31(4):573-575. DOI: 10.4103/09709185.169101. 